\title{
NULL FINITE TYPE HYPERSURFACES IN SPACE FORMS
}

\author{
By A. FerrándeZ* AND P. LUCAS**
}

\section{Introduction}

In [5], Chen gives a classification of null 2-type surfaces in the Euclidean 3-space and he shows in [6] that a similar characterization cannot be given for a surface in the Euclidean 4-space. In fact, helical cylinders in Euclidean 4space are characterized as those surfaces of null 2-type and constant mean curvature.

In this paper we give a characterization of null 2-type hypersurfaces in a space of constant sectional curvature $\bar{M}^{n+1}(k)$ and an approach to hypersurfaces of null 3-type. Indeed, we get a generalization of Chen's paper [5] not only by considering hypersurfaces, but also taking them in space forms.

In spherical and hyperbolic cases we show that there is no null 2-type hypersurface, so that the Euclidean case becomes the most attractive situation where our classification works on. Actually, we show that Euclidean hypersurfaces of null 2-type and having at most two distinct principal curvatures are locally isometric to a generalized cylinder. Why the hypothesis on principal curvatures? First, we think this is the most natural one, because, after Chen's paper, we know that cylinders are the only surfaces of null 2-type in Euclidean 3-space. Secondly, it is well-known that a Euclidean isoparametric hypersurface has at most two distinct principal curvatures, so that if it has exactly two, then one of them has to be zero. Our classification depends strongly on that isoparametricity condition. Finally, bounding the number of principal curvatures is not as restrictive as one could hope. As a matter of fact, the families of conformally flat and rotational hypersurfaces satisfy that hypothesis and both are sufficiently large so that it is worth trying to give a characterization of some subfamily of them in order to get along in their classifications. To this effect, we characterize rotational and conformally flat hypersurfaces of null 2type.

As for hypersurfaces of null 3-type one immediately sees that they are not difficult to handle when they have constant mean curvature, because a nice

* Partially supported by a DGICYT Grant No. PS87-0115-C03-03.

** Supported by a FPPI Grant, Program PG, Ministerio de Educatión y Ciencia.

Received August 7, 1990; revised March 5, 1991. 
formula for $\Delta^{2} H$ can be given. In that case, we show that there is no spherical or hyperbolic hypersurface of null 3-type. It turns out again that our only hope to get some more information concerns with Euclidean hypersurfaces. Now, following a similar reasoning as in the null 2-type case, we are able to say that there is no Euclidean hypersurface of null 3-type having constant mean curvature and at most two distinct principal curvatures.

We wish to thank to Prof. M. Barros for many valuable comments and suggestions.

\section{Preliminaries}

Let $x: M^{n} \rightarrow \boldsymbol{R}^{m}$ be an isometric immersion of a connected $n$-dimensional Riemannian manifold $M$ into the Euclidean space $\boldsymbol{R}^{m}$. We represent by $\Delta$ the Laplacian operator of $M$ (with respect to the induced metric) acting on the space of smooth functions $\mathcal{C}^{\infty}(M)$. The manifold $M$ is said to be of $k$-type if the position vector $x$ of $M$ can be decomposed in the following form:

where

$$
x=x_{0}+x_{\imath_{1}}+\cdots+x_{\imath_{k}},
$$

$$
\Delta x_{i}=\lambda_{i_{j}} x_{i_{j}}
$$

$\lambda_{i_{1}}<\cdots<\lambda_{i_{k}}, x_{0}$ is a constant vector in $\boldsymbol{R}^{m}$ (when $M$ is compact, $x_{0}$ is the center of mass of $M$ in $\boldsymbol{R}^{m}$ ) and $\Delta$ is the extension of the Laplace operator to $\boldsymbol{R}^{m}$-valuated smooth functions on $M$ in a natural way. A manifold $M$ is said to be of finite type if it is of $k$-type for some natural number $k$; otherwise, $M$ is said to be of infinite type. A special case appears when some $\lambda_{\imath}=0$; then $M$ is said to be of null $k$-type or null finite type.

If $M$ is of finite type, for example of $k$-type, from (1.1) there exists a monic polynomial, say $Q(t)$, such that $Q(\Delta)\left(x-x_{0}\right)=0$. If we suppose that $Q(t)$ $=t^{k}+d_{1} t^{k-1}+\cdots+d_{k-1} t+d_{k}$ then coefficients $d_{\imath}$ are given by

$$
d_{1}=-\sum_{t=1}^{k} \lambda_{i_{t}} ; \quad d_{2}=\sum_{t<t^{\prime}} \lambda_{i_{t}} \lambda_{\imath_{t^{\prime}}} ; \cdots d_{k}=(-1)^{k} \lambda_{\imath_{1}} \lambda_{2_{2}} \cdots \lambda_{2_{k}},
$$

where $\left\{\lambda_{i_{1}}, \cdots, \lambda_{\imath_{k}}\right\}$ are the associated eigenvalues giving the $k$-type character. Therefore, by the formula $\Delta x=-n H$, where $H$ is the mean curvature vector field of $M$ in $\boldsymbol{R}^{m}$, we have the following differential equation:

$$
\Delta^{k-1} H+d_{1} \Delta^{k-2} H+\cdots+d_{k-1} H-\frac{d_{k}}{n}\left(x-x_{0}\right)=0 .
$$

We note that $d_{k}=0$ when $M$ is of null $k$-type and therefore (1.3) only contains terms involving the mean curvature vector $H$.

Let $\boldsymbol{R}_{s}^{m}$ be the $m$-dimensional pseudo-Euclidean space with the standard flat pseudo-Riemannian metric of signature $(s, m-s)$ and $x: M^{n} \rightarrow \boldsymbol{R}_{s}^{m}$ an isometric immersion of a connected pseudo-Riemannian (or space-like) submanifold $M$ in 
$\boldsymbol{R}_{s}^{m}$. Then, in this context, we can introduce the finite type notion in a similar way as in the Euclidean case. Thus we can characterize the pseudo-Riemannian (or space-like) submanifolds of $k$-type by equation (1.3), in this case $H$ is the mean curvature vector field of $M$ in $\boldsymbol{R}_{s}^{m}$. For the general knowledge on Finite Type Submanifolds in pseudo-Euclidean spaces, see for instance $[3,4]$.

\section{The spherical case}

Let $M^{n}$ be a hypersurface in the unit sphere $S^{n+1}$ centred at the origin of $\boldsymbol{R}^{n+2}$. Let us denote by $x: M^{n} \rightarrow S^{n+1} \subset \boldsymbol{R}^{n+2}$ the immersion and by $\nabla, D, D^{\prime}$ the Riemannian connection of $M$, the normal connection of $M$ in $\boldsymbol{R}^{n+2}$ and the normal connection of $M$ in $S^{n+1}$, respectively. Let $\sigma$ and $H$ (resp. $\sigma^{\prime}$ and $H^{\prime}$ ) be the second fundamental form and the mean curvature vector of $M$ in $\boldsymbol{R}^{n+2}$ (resp. of $M$ in $S^{n+1}$ ) and let $A$ be the Weingarten map of $M$ in $S^{n+1}$. Then from [1, Lemma 4] we have the following expression of $\Delta H$ :

$$
\Delta H=\Delta^{D^{\prime}} H^{\prime}+\frac{n}{2} \nabla \alpha^{2}+2 \operatorname{tr} A_{D H^{\prime}}+|\sigma|^{2} H^{\prime}-n \alpha^{2} x,
$$

where $\alpha^{2}=\langle H, H\rangle$ and $\operatorname{tr} A_{D H^{\prime}}=$ trace $\left\{(X, Y) \rightarrow A_{D_{X} H^{\prime}} Y\right\}$. Assume now $M$ is of 2-type, i.e., $\Delta H=b H+c\left(x-x_{0}\right)$. Let $f: M \rightarrow \boldsymbol{R}$ be the function defined by $f(x)$ $=\left\langle x, x_{0}\right\rangle$. Then for any tangent vector field $X$ to $M$, we have

$$
\left\langle(\Delta H)^{T}, X\right\rangle=-c X(f),
$$

where $(\Delta H)^{T}$ means the tangent component of $\Delta H$ in $M$. Then

$$
(\Delta H)^{T}=-c \nabla f .
$$

By using (2.1) one gets

$$
\langle\Delta H, x\rangle=-n \alpha^{2}
$$

and again from the 2-type condition we have

$$
\langle\Delta H, x\rangle=-b+c-c f .
$$

From (2.3) and (2.4) we deduce

that jointly with (2.2) yield to

$$
-c \nabla f=-n \nabla \alpha^{2}
$$

$$
(\Delta H)^{T}=-n \nabla \alpha^{2} .
$$

On the other hand, since $\Delta^{D^{\prime}}$ denotes the Laplacian associated with $D^{\prime}$ and one has the formula

$$
\Delta H^{\prime}=\left(\Delta \alpha^{\prime}\right) N+\alpha^{\prime} \Delta N+2 A\left(\nabla \alpha^{\prime}\right),
$$

where $H^{\prime}=\alpha^{\prime} N$ and $A=A_{N}$, then 


$$
\Delta^{D^{\prime}} H^{\prime}=\left(\Delta \alpha^{\prime}\right) N
$$

The above formulae allow us to write down

$$
\Delta H=-n \nabla \alpha^{2}+\left(\Delta \alpha^{\prime}\right) N+|\sigma|^{2} H^{\prime}-n \alpha^{2} x .
$$

At this point, the 2-type condition can be rewritten in the following useful form

$$
c\left(x-x_{0}\right)=-n \nabla \alpha^{2}+\left\{\Delta \alpha^{\prime}+|\sigma|^{2} \alpha^{\prime}-b \alpha^{\prime}\right\} N+\left\{b-n \alpha^{2}\right\} x .
$$

In [1], studying the compact case with the additional condition of having at most two distinct principal curvatures, it is shown that $M$ is of 2-type if and only if it is a product of two spheres with appropriate radii. In our case, if we drop the compactness condition, then one of the eigenvalues could vanish, but that can not hold as the following result shows, which can also be deduced from [7, Theorem 1$]$.

PROPOSITION 2.1. There is no spherical hypersurface of null 2-type.

Proof. If $M$ is a spherical hypersurface of null 2-type, then $c=0$. Now from (2.9), the $x$-component vanishes and then $\alpha$ is constant. Since $M$ is not minimal, from the $N$-component, we have that $|\sigma|^{2}$ is also constant. Using again (2.9), one gets $|\sigma|^{2}=n \alpha^{2}$, which is a contradiction with the following lemma and the proof finishes.

Lemma 2.2. Let $M$ be a spherical hypersurface. Then the following inequality holds

$$
n \alpha^{2} \leqq|\sigma|^{2},
$$

and the equality holds if and only if $M$ is of 1-type.

Proof. Let us denote by $\mu_{1}, \cdots, \mu_{n}$ the principal curvatures of $M$ and take the vectors $V=\left(\mu_{1}, \cdots, \mu_{n}\right)$ and $W=(1, \cdots, 1)$. Then from the own definitions of $\alpha^{\prime}$ and $\left|\sigma^{\prime}\right|^{2}$ and the Cauchy-Schwarz inequality the first part of lemma follows. Moreover, the equality holds if and only if $M$ is totally umbilical and therefore of 1-type.

In the realm of finite type submanifolds, as far as we know, the 3-type case has been rather scarcely studied. This is because it is difficult to find a nice expression for $\Delta^{2} H$. Therefore, we shall assume that $M$ is of constant mean curvature.

Then, a straightforward computation from (2.8) yields

$$
\begin{aligned}
\Delta^{2} H= & 2 \alpha^{\prime} A\left(\nabla|\sigma|^{2}\right)+\left\{\Delta|\sigma|^{2}+|\sigma|^{4}-n|\sigma|^{2}+n^{2} \alpha^{2}\right\} H^{\prime} \\
& -\left\{n|\sigma|^{2} \alpha^{\prime 2}+n^{2} \alpha^{2}\right\} x .
\end{aligned}
$$

Now, if $M$ is of 3 -type, i.e., $\Delta^{2} H=a \Delta H+b H+c\left(x-x_{0}\right)$, we have 


$$
\begin{aligned}
c\left(x-x_{0}\right)= & 2 \alpha^{\prime} A\left(\nabla|\sigma|^{2}\right) \\
& +\left\{\Delta|\sigma|^{2}+|\sigma|^{4}-n|\sigma|^{2}+n^{2} \alpha^{2}-a|\sigma|^{2}-b\right\} H^{\prime} \\
& -\left\{n|\sigma|^{2} \alpha^{\prime 2}+n^{2} \alpha^{2}-a n \alpha^{2}-b\right\} x .
\end{aligned}
$$

We are ready to give another non-existence result.

PROPOSITION 2.3. There is no spherical hypersurface of null 3-type with constant mean curvature.

Proof. If $M$ is a spherical hypersurface of null 3-type, then $c=0$. Since the mean curvature $\alpha^{\prime}$ of $M$ is a non-vanishing constant, from the $x$-component in (2.11), one has $|\sigma|^{2}$ is also a constant. Then, once more from (2.11), we get

$$
n|\sigma|^{2}-n^{2} \alpha^{2}+b=|\sigma|^{2}\left(|\sigma|^{2}-a\right)=n \alpha^{2}\left(|\sigma|^{2}-a\right),
$$

from which we deduce

$$
\left(|\sigma|^{2}-n \alpha^{2}\right)\left(|\sigma|^{2}-a\right)=0 .
$$

But $|\sigma|^{2}=n \alpha^{2}$ can not hold from Lemma 2.2. If $|\sigma|^{2}=a>0$, from (2.12) and Lemma 2.2 it follows that $b<0$. Then we can find a real number $p \neq 0$, (indeed, $p$ is a solution of the equation $p^{2}-a p-b=0$ ) such that if we write

$$
\begin{aligned}
& y_{1}=\frac{b}{p^{2}+b} x+\frac{p}{p^{2}+b} \Delta x, \\
& y_{2}=\frac{p^{2}}{p^{2}+b} x-\frac{p}{p^{2}+b} \Delta x,
\end{aligned}
$$

we have

where

$$
x=y_{1}+y_{2},
$$

$$
\begin{gathered}
\Delta y_{1}=p y_{1}, \\
\Delta y_{2}=-\frac{b}{p} y_{2},
\end{gathered}
$$

showing that $M$ is of 2 -type, which is a contradiction.

\section{The Euclidean case}

In order to make a similar study to the spherical case, we start giving a formula for $\Delta H$, which for surfaces was found by B. Y. Chen in [5].

LEMMA 3.1 Let $x: M^{n} \rightarrow \boldsymbol{R}^{n+1}$ be an orientable Euclidean hypersurface. Then

$$
\Delta H=2 A(\nabla \alpha)+\frac{n}{2} \nabla \alpha^{2}+\left\{\Delta \alpha+\alpha|A|^{2}\right\} N,
$$


where $H=\alpha N,, N$ being a global unit normal vector field.

Proof. Let $p$ be in $M,\left\{E_{1}, \cdots, E_{n}\right\}$ a local orthonormal frame tangent to $M$ such that $\nabla_{E_{i}} E_{j}(p)=0$ and $\bar{\nabla}$ the connection in $\boldsymbol{R}^{n+1}$. From the following formulae

$$
\begin{gathered}
\bar{\nabla}_{E_{i}} H=E_{i}(\alpha) N-\alpha A E_{\imath}, \\
\bar{\nabla}_{E_{\imath}} \bar{\nabla}_{E_{i}} H=E_{\imath} E_{i}(\alpha) N-2 E_{i}(\alpha) A E_{i}-\alpha\left\{\left(\nabla_{E_{i}} A\right) E_{i}+\sigma\left(A E_{\imath}, E_{\imath}\right)\right\},
\end{gathered}
$$

we have

$$
\Delta H=2 A(\nabla \alpha)+\alpha \operatorname{tr}(\nabla A)+\left\{\Delta \alpha+\alpha|A|^{2}\right\} N,
$$

where $\operatorname{tr}(\nabla A)=\sum_{\imath=1}^{n}\left(\nabla_{E_{i}} A\right) E_{\imath}$.

To compute $\operatorname{tr}(\nabla A)$, let $\left\{X_{1}, \cdots, X_{n}\right\}$ be the local orthonormal frame of eigenvectors of the Weingarten map, i.e., $A X_{\imath}=\mu_{\imath} X_{\imath}$. Then, using the wellknown equations

$$
\nabla_{X_{i}} X_{J}=\sum_{k=1}^{n} \omega_{j}^{k}\left(X_{\imath}\right) X_{k}
$$

we have

$$
\operatorname{tr}(\nabla A)=\sum_{j=1}^{n} X_{j}\left(\mu_{\jmath}\right) X_{j}+\sum_{\imath, j}\left(\mu_{i}-\mu_{\jmath}\right) \omega_{i}^{\jmath}\left(X_{\imath}\right) X_{\jmath} .
$$

Now, from Codazzi's equation $\left(\nabla_{X_{i}} A\right) X_{j}=\left(\nabla_{X_{j}} A\right) X_{\imath}$, one gets

Then

$$
X_{j}\left(\mu_{i}\right)=\left(\mu_{i}-\mu_{j}\right) \omega_{i}^{\top}\left(X_{\imath}\right) \text {. }
$$

$$
\operatorname{tr}(\nabla A)=n \nabla \alpha
$$

and the lemma follows from here and (3.1).

Now, if $M$ is of 2-type, i.e., $\Delta H=b H+c x$ (where we assume without loss of generality that $x_{0}$ is the origin of $\boldsymbol{R}^{n+1}$ ), from the above lemma we have

$$
c x=2 A(\nabla \alpha)+\frac{n}{2} \nabla \alpha^{2}+\left\{\Delta \alpha+\alpha|A|^{2}-b \alpha\right\} N .
$$

This formula allow us to get three easy and interesting consequences, chiefly the third because it will be very useful through this section. The first and second ones have already been obtained by Chen and Lue in [7].

COROLLARY 3.2. If $M$ is a non minimal Euclidean hypersurface of at most 2- type and constant mean curvature, then one of the two following possibilities holds :

(i) $M$ is of null 2-type,

(ii) $M$ is an open piece of $S^{n}$.

COROLLARY 3.3. If $M$ is a compact Euclidean hypersurface of at most 2- 
type, then $M$ has constant mean curvature if and only if $M$ is isometric to sphere $S^{n}$.

Remark 3.4. The above corollary shows that there is no compact hypersurface of 2-type having constant mean curvature.

COROLlaRY 3.5. If $M$ is a Euclidean hypersurface of null 2-type, then

$$
A\left(\nabla \alpha^{2}\right)=-\frac{n}{2} \alpha \nabla \alpha^{2}
$$

The problem of characterizing Euclidean hypersurfaces of null 2-type does not seem an easy task without additional hypothesis and actually it is more difficult than spherical case (see Proposition 2.1). The constancy of the mean curvature does not even provide, in principle, enough information to get such characterization. Nevertheless, we have the following result.

PROPOSITION 3.6. Let $M$ be a Euclidean hypersurface with at most two principal curvatures. Then $M$ is of null 2-type and constant mean curvature if and only if it is locally isometric to a product $\boldsymbol{R}^{p} \times S^{n-p}(r)$.

Proof. If $M$ is of null 2-type and has constant mean curvature, by using (3.5) we have $|A|^{2}$ is a constant. Furthermore, the hypothesis on principal curvatures yields to $M$ has exactly two constant principal curvatures. From [10] $M$ is an open piece of $\boldsymbol{R}^{p} \times S^{n-p}(r)$. The converse is trivial.

In the proof of the above proposition, to use the Segre's result, it has been crucial to deduce that $M$ is isoparametric and for that to be possible we have needed the hypothesis on principal curvatures. The isoparametricity condition on $M$ is rather strong and owing to the recent results showing the close relation between Dupin and isoparametric hypersurfaces, one can get another approximation to characterize null 2-type hypersurfaces.

Proposition 3.7. If $M$ is a Dupin Euclidean hypersurface of null 2-type, then $M$ has constant mean curvature.

Proof. If the mean curvature $\alpha$ were not constant, from Corollary 3.5, at the points of the open set $\mathcal{V}=\left\{p \in M: \nabla \alpha^{2}(p) \neq 0\right\}$, we can choose a local orthonormal frame $\left\{E_{1}, \cdots, E_{n}\right\}$ tangent to $M$ such that $E_{1}$ is parallel to $\nabla \alpha^{2}$. That means $E_{2}(\alpha)=\cdots=E_{n}(\alpha)=0$. Since $M$ is a Dupin hypersurface and $E_{1}$ is a principal direction with principal curvature $-(n / 2) \alpha$, then $E_{1}(\alpha)=0$, which is a contradiction.

COROLlary 3.8. Let $M$ be a Dupin Euclidean hypersurface with at most two principal curvatures. Then $M$ is of null 2-type if and only if $M$ is locally isometric to a product $\boldsymbol{R}^{p} \times S^{n-p}(r)$. 
To get down to work in a more general situation we need a previous lemma.

LEMma 3.9. Let $M$ be a Euclidean hypersurface of null 2-type with at most two principal curvatures. Then $Q V$ is empty or, at the points of $C V,-(n / 2) \alpha$ is a principal curvature with multiplicity one.

Proof. At the points of $\mathcal{C}$, by using Corollary $3.5,-(n / 2) \alpha$ is a principal curvature with associated principal direction $\nabla \alpha^{2}$. Let $V_{1}$ be the open subset of $Q$ where the mean curvature does not vanish. Then $V_{1}$ is not empty, if $Q V$ is not, and on $V_{1}$ there are exactly two distinct principal curvatures. Choose the local orthonormal frame $\left\{E_{1}, \cdots, E_{n}\right\}$ of principal directions such that $E_{1}$ is parallel to $\nabla \alpha^{2}$. Let $D=\{X \in T \subset V: A X=-(n / 2) \alpha X\}$ be the distribution assocoiated with the eigenvalue $-(n / 2) \alpha$, which is differentiable and involutive in the open set $V_{1}$. If we assume $\operatorname{dim} D>1$, from [9, Proposition 2.3], we have $X(-(n / 2) \alpha)=0$ for any vector field $X \in D$. In particular, $E_{1}(\alpha)=0$ on $V_{1}$, so that being $E_{1}$ and $\nabla \alpha^{2}$ parallel, we get $\alpha$ is a constant on $V_{1}$, which is a contradiction. Therefore, $\operatorname{dim} D=1$ and $-(n / 2) \alpha$ has multiplicity one.

Now, the main result of this section states as follows.

THEOREM 3.10. Let $M$ be a Euclidean hypersurface with at most two principal curvatures. Then $M$ is of null 2-type if and only if $M$ is locally isometric to a product $\boldsymbol{R}^{p} \times S^{n-p}(r)$.

Proof. Suppose $M^{n}$ is a Euclidean hypersurface. Our goal is to prove that $M^{n}$ has constant mean curvature. If $\alpha$ were not constant, then by the Lemma $3.9 C V$ is not empty and the vector $\nabla \alpha^{2}$ is an eigenvector of $A$ corresponding to the eigenvalue $-(n / 2) \alpha$ with multiplicity 1 . Choose a local orthonormal frame $\left\{E_{1}, \cdots, E_{n}\right\}$, in an open set of $\mathcal{C}$, satisfying that $\left\{E_{1}, \cdots, E_{n}\right\}$ are eigenvectors of $A$ and $E_{1}$ is parallel to $\nabla \alpha^{2}$.

Now by hypothesis $\Delta H=b H$ so that from Lemma 3.1 we have

$$
\Delta^{D} H=\left(b-|A|^{2}\right) H ; A(\nabla \alpha)+\frac{n}{2} \alpha \nabla \alpha=0 .
$$

Let $\left\{\omega^{1}, \cdots, \omega^{n}\right\}$ and $\left\{\omega_{i}^{j}\right\}, i, j=1, \cdots, n+1$, the dual frame and the connection forms of the chosen frame. Then we have

$$
\begin{aligned}
\omega_{n+1}^{1}=\frac{n}{2} \alpha \omega^{1} ; \quad \omega_{n+1}^{3} & =-\frac{3}{2} \frac{n}{n-1} \alpha \omega^{j}, \quad j=2, \cdots, n . \\
d \alpha & =E_{1}(\alpha) \omega^{1} .
\end{aligned}
$$

From the first equation of (3.7) we have

$$
d \omega_{n+1}^{1}=\frac{n}{2} \alpha d \omega^{1} .
$$


Using now the second equation of (3.7) and the structure equations, one has

$$
d \omega_{n+1}^{1}=-\frac{3}{2} \frac{n}{n-1} \alpha d \omega^{1} .
$$

These two last equations mean that

$$
d \omega^{1}=0 .
$$

Therefore one locally has $\omega^{1}=d u$, for a certain function $u$, which along with (3.8) imply that $d \alpha \wedge d u=0$. Thus $\alpha$ depends on $u, \alpha=\alpha(u)$. Then $d \alpha=\alpha^{\prime} d u=$ $\alpha^{\prime}(u) \omega^{1}$ and so $E_{1}(\alpha)=\alpha^{\prime}$.

Taking differentiation in the second equation of (3.7) we have

$$
d \omega_{n+1}^{\jmath}=-\frac{3}{2} \frac{n}{n-1} \alpha^{\prime} \omega^{1} \wedge \omega^{\jmath}-\frac{3}{2} \frac{n}{n-1} \alpha d \omega^{\jmath},
$$

and, also by the structure equations :

$$
d \omega_{n+1}^{3}=-\frac{3}{2} \frac{n}{n-1} \alpha d \omega^{\jmath}-\frac{n(n+2)}{2(n-1)} \alpha \omega_{1}^{\jmath} \wedge \omega^{1} .
$$

Consequently

$$
\omega_{j}^{1}=\frac{3}{n+2} \frac{\alpha^{\prime}}{\alpha} \omega^{j}, \quad j=2, \cdots, n,
$$

that is

$$
(n+2) \alpha \omega_{j}^{1}=3 \alpha^{\prime} \omega^{j}, \quad j=2, \cdots, n .
$$

Differentiating (3.15) and using (3.7) and (3.14) we have

$$
\begin{gathered}
d\left(\alpha \omega_{j}^{1}\right)=\frac{3}{n+2} \frac{\left(\alpha^{\prime}\right)^{2}}{\alpha} \omega^{1} \wedge \omega^{j}+\alpha d \omega_{j}^{1}, \\
d \omega_{j}^{1}=-\frac{3}{4} \frac{n^{2}}{n-1} \alpha^{2} \omega^{1} \wedge \omega^{j}+\frac{3}{n+2} \frac{\alpha^{\prime}}{\alpha}\left(d \omega^{j}+\frac{3}{n+2} \frac{\alpha^{\prime}}{\alpha} \omega^{1} \wedge \omega^{j}\right) .
\end{gathered}
$$

On the other hand

$$
d\left(\alpha^{\prime} \omega^{j}\right)=\alpha^{\prime \prime} \omega^{1} \wedge \omega^{j}+\alpha^{\prime} d \omega^{j} .
$$

Hence from (3.15) to (3.18) we obtain

$$
4 \alpha \alpha^{\prime \prime}-\frac{4(n+5)}{n+2}\left(\alpha^{\prime}\right)^{2}+\frac{n^{2}(n+2)}{n-1} \alpha^{4}=0 .
$$

Putting $y=\left(\alpha^{\prime}\right)^{2}$ the above equation turns into

$$
2 \frac{\alpha}{\alpha^{\prime}} y^{\prime}-\frac{4(n+5)}{n+2} y=-\frac{n^{2}(n+2)}{n-1} \alpha^{4},
$$

and then 


$$
y=\left(\alpha^{\prime}\right)^{2}=C \alpha^{2(n+5) / n+2}-\left(\frac{n(n+2)}{2(n-1)}\right)^{2} \alpha^{4},
$$

with $C$ a constant.

Now we use the definition of $\Delta \alpha$, the fact that $E_{1}$ is parallel to $\nabla \alpha^{2}$ and equation (3.14) to obtain

$$
(n+2) \alpha \Delta \alpha=-(n+2) \alpha \alpha^{\prime \prime}+3(n-1)\left(\alpha^{\prime}\right)^{2} .
$$

As we know $\Delta^{D} H=(\Delta \alpha) N$, hence from (3.6) we get

$$
\alpha \Delta \alpha=\left(b-|A|^{2}\right) \alpha^{2} .
$$

Since $|A|^{2}=\left(n^{2}(n+8) / 4(n-1)\right) \alpha^{2}$, combining (3.22) and (3.23), we have

$$
\alpha \alpha^{\prime \prime}-\frac{3(n-1)}{(n+2)}\left(\alpha^{\prime}\right)^{2}+\left(b-\frac{n^{2}(n+8)}{4(n-1)} \alpha^{2}\right) \alpha^{2}=0 .
$$

Thus, putting together (3.19) and (3.24) one has

$$
\frac{2(n-4)}{n+2}\left(\alpha^{\prime}\right)^{2}=b \alpha^{2}-\frac{n^{2}(n+5)}{2(n-1)} \alpha^{4} .
$$

We deduce, using (3.21) and (3.25) that $\alpha$ is locally constant on $\mathcal{C}$, which is a contradiction with the definition of $\mathcal{C}$. Hence $\alpha$ is constant on $M^{n}$ and the result follows from Proposition 3.6. The converse is trivial and the proof finishes.

Then we obtain the following consequence, which B.Y. Chen already gave in $[5]$.

COROllary 3.11. Let $M$ be a surface in $\boldsymbol{R}^{3}$. Then $M$ is of null 2-type if and only if $M$ is locally isometric to a circular cylinder.

A we did in the spherical case, we now approach the Euclidean hypersurfaces of null 3-type with the additional assumption of having constant mean curvature. Then a direct computation from Lemma 3.1 yields to

$$
\Delta^{2} H=2 \alpha A\left(\nabla|A|^{2}\right)+\left\{\Delta|A|^{2}+|A|^{4}\right\} H .
$$

Thus, if $M$ is of 3 -type, we have

$$
c x=2 \alpha A\left(\nabla|A|^{2}\right)+\left\{\Delta|A|^{2}+|A|^{4}-a|A|^{2}-b\right\} H,
$$

and then we obtain the following

PROPOSITION 3.12. There is no Euclidean hypersurface of null 3-type with constant mean curvatnre and having at most two distinct principal curvatures.

Proof. Let $M$ be a Euclidean hypersurface of null 3-type. From (3.27), since $\alpha$ is a non vanishing constant, we have $A\left(\nabla|A|^{2}\right)=0$. If we consider the open set $W=\left\{p \in M: \nabla|A|^{2}(p) \neq 0\right\}$, then at the points of $W, \nabla|A|^{2}$ is a principal 
direction with 0 principal curvature. Therefore, since $M$ has at most two principal curvatures and $\alpha$ is constant, if $\lambda$ is the other principal curvature, then $\lambda$ is also a constant. Then $M$ is isoparametric and so it can not be of null 3-type.

\section{The hyperbolic case}

Let $\boldsymbol{R}_{1}^{n+2}$ the $(n+2)$-dimensional pseudo-Euclidean space with metric tensor given by

$$
\langle,\rangle=-d x^{1} \otimes d x^{1}+\sum_{j=2}^{n+2} d x^{j} \otimes d x^{\jmath},
$$

where $\left(x_{1}, \cdots, x_{n+2}\right)$ is a rectangular coordinate system in $\boldsymbol{R}_{1}^{n+2}$. Then $\left(\boldsymbol{R}_{1}^{n+2}\right.$, $<,>)$ is a flat pseudo-Riemannian manifold with signature $(1, n+1)$. We define

$$
\left.H^{n+1}(R)=\left\{x \in \boldsymbol{R}_{1}^{n+2}:\langle x, x\rangle=-R^{2}, x_{1}\right\rangle 0\right\},
$$

and $H^{n+1}=H^{n+1}(1)$ the $(n+1)$-dimensional hyperbolic space.

Let $M^{n}$ be a hypersurface in $H^{n+1}$ and denote by $x: M^{n} \rightarrow H^{n+1} \subset \boldsymbol{R}_{1}^{n+2}$ the natural immersion. We now use the same notation as in Section 2, where the symbols concerning there with $S^{n+1}$ (resp. $\boldsymbol{R}^{n+2}$ ), will be here the corresponding for $H^{n+1}$ (resp. $\boldsymbol{R}_{1}^{n+2}$ ).

We start with a useful expression for $\Delta H$, which essentially is given in [3].

LEMMA 4.1. Let $M$ be a hypersurface in $H^{n+1}$. Then

$$
\Delta H=2 A\left(\nabla \alpha^{\prime}\right)+\frac{n}{2} \nabla \alpha^{\prime 2}+\left(\Delta \alpha^{\prime}\right) N+\left(|A|^{2}-n\right) H^{\prime}+\left(n \alpha^{\prime 2}-n\right) x .
$$

Proof. It suffices to work as in the Euclidean case (Lemma 3.1) taking into account the following facts:

$$
\begin{gathered}
H=H^{\prime}+x, \\
A_{x}=-I, \\
\sigma\left(A E_{\imath}, E_{i}\right)=\left\langle\sigma\left(A E_{\imath}, E_{i}\right), N\right\rangle N-\left\langle\sigma\left(A E_{\imath}, E_{\imath}\right), x\right\rangle x .
\end{gathered}
$$

If $M$ is of 2 -type in $\boldsymbol{R}_{1}^{n+2}$, then from Lemma 4.1 we get

$$
\begin{aligned}
c\left(x-x_{0}\right)= & 2 A\left(\nabla \alpha^{\prime}\right)+\frac{n}{2} \nabla \alpha^{\prime 2}+\left\{\Delta \alpha^{\prime}+\alpha^{\prime}|A|^{2}-n \alpha^{\prime}-b \alpha^{\prime}\right\} N \\
& +\left\{n \alpha^{\prime 2}-n-b\right\} x,
\end{aligned}
$$

where, as above, $\Delta H=b H+c\left(x-x_{0}\right)$.

As an example of a hyperbolic hypersurface of 2 -type in $\boldsymbol{R}_{1}^{n+2}$ we define

$$
H^{n-k}\left(\sqrt{1+r^{2}}\right) \times S^{k}(r)=\left\{x \in H^{n+1}: x_{n-k+1}^{2}+\cdots+x_{n+2}^{2}=r^{2}\right\} .
$$

If we put $y_{1}=\left(x_{1}, x_{2}, \cdots, x_{n-k}, 0, \cdots, 0\right)$ and $y_{2}=\left(0, \cdots, 0, x_{n-k+1}, \cdots, x_{n+2}\right)$, it 
is easy to see that $x=y_{1}+y_{2}$ where $\Delta y_{1}=\left(-(n-k) / 1+r^{2}\right) y_{1}$ and $\Delta y_{2}=\left(k / r^{2}\right) y_{2}$, so that $x$ is of 2-type in $\boldsymbol{R}_{1}^{n+2}$.

However, for the null 2-type case we have

Proposition 4.2. There is no hyperbolic hypersurface of null 2-type.

Proof. If $M$ is a hyperbolic hypersurface of null 2-type, then from (4.1) both $\alpha^{\prime}$ and $|A|^{2}$ are constant and furthermore $|A|^{2}=n \alpha^{\prime 2}$. This will mean that $M$ is totally umbilical and then of 1-type, which is a contradiction.

To finish this section we shall study the hyperbolic hypersurfaces of 3-type with constant mean curvature. Indeed, a straightforward computation from Lemma 4.1 gives

$$
\begin{gathered}
\Delta^{2} H=2 \alpha^{\prime} A\left(\nabla|A|^{2}\right)+\left\{\Delta|A|^{2}+|A|^{4}-|A|^{2} n-n^{2} \alpha^{\prime 2}+n^{2}\right\} H^{\prime} \\
+\left\{n \alpha^{\prime 2}|A|^{2}-2 n^{2} \alpha^{\prime 2}+n^{2}\right\} x .
\end{gathered}
$$

When $M$ is of 3-type, we have

$$
\begin{aligned}
c\left(x-x_{0}\right)= & 2 \alpha^{\prime} A\left(\nabla|A|^{2}\right) \\
& +\left\{\Delta|A|^{2}+|A|^{4}-|A|^{2} n-n^{2} \alpha^{2}+n^{2}-a\left(|A|^{2}-n\right)-b\right\} H^{\prime} \\
& +\left\{n \alpha^{\prime 2}|A|^{2}-2 n^{2} \alpha^{\prime 2}+n^{2}-a\left(n \alpha^{\prime 2}-n\right)-b\right\} x .
\end{aligned}
$$

Therefore, in a similar way as in the spherical case, we have

Proposition 4.3. There is no hyperbolic hypersurface of null 3-type with constant mean curvature.

\section{Conclusions}

Now, we are going to get together in a more general situation the results we have get in the above sections.

MAIN THEOREM. Let $M^{n}$ be a hypersurface in a space form $\bar{M}^{n+1}(k)$. Then we have:

(i) If $M$ is of null 2-type, then $k=0$. Moreover, if $M$ has at most two distinct principal curvatures, then $M$ is locally isometric to a product $\boldsymbol{R}^{n-k} \times S^{k}(r)$.

(ii) Assume that one of the following assertions holds:

1) $k \neq 0$,

2) $k=0$ and $M$ has at most two distinct principal curvatures.

Then either $M$ has non constant mean curvature or $M$ is not of null 3-type.

Now, we can deduce a few interesting consequences.

In [2] it is shown that a rotational hypersurface in a space of constant 
curvature has two principal curvatures, one of them having multiplicity at least equal to $n-1$. Therefore, we have

COROLlARY 5.1. Let $x: M^{n} \rightarrow \bar{M}^{n+1}(k)$ be a rotational hypersurface of null 2-type. Then $k=0$ and $M$ is locally isometric to a product $\boldsymbol{R} \times S^{n-1}(r)$.

It is a well-known fact (see [8]) that, for $n>3$, a hypersurface $M^{n}$ in $\bar{M}^{n+1}(k)$ is conformally flat if and only if at least $n-1$ principal curvatures are all equal. Then we get

COROLlARY 5.2. Let $x: M^{n} \rightarrow \bar{M}^{n+1}(k), n>3$, be a conformally flat hypersurface of null 2-type. Then $k=0$ and $M$ is locally isometric to a product $\boldsymbol{R} \times$ $S^{n-1}(r)$ or $\boldsymbol{R}^{n-1} \times S^{1}(r)$.

If $M$ is an Einstein hypersurface, we know $M$ has at most two distinct principal curvatures. Then from [9, Theorem 3.1] we have

COROLLARY 5.3. Let $x: M^{n} \rightarrow \bar{M}^{n+1}(k), n>2$, be an Einstein hypersurface of null 2-type. Then $k=0$ and $M$ is locally isometric to $R^{n-1} \times S^{1}(r)$.

Added in proof. After this paper was referred, prof. Chen kindly pointed out to us that himself and S. J. Li have shown, in a joint paper, that Proposition 2.3 holds for every 3-type spherical hypersurface. We wish to thank to the referee and prof. Chen for their careful suggestions in order to improve this article.

\section{REFERENCES}

[1] M. Barros, B. Y. Chen and O.J. Garay, Spherical finite type hypersurfaces, Algebras, Groups and Geometries 4 (1987), 58-72.

[2] M. Do CARmo AND M. DAJCzer, Rotation hypersurfaces in spaces of constant curvature, Transactions of the A.M.S. 277 (1983), 685-709.

[3] B. Y. CHEN, Finite type submanifolds in pseudo-Euclidean spaces and applications, Kodai Math. J. 8 (1985), 358-374.

[4] B. Y. CHEN, Finite-type pseudo-Riemannian submanifolds, Tamkang J. of Math. 17 (2) (1986), 137-151.

[5] B. Y. CHEN, Null 2-type surfaces in $E^{3}$ are circular cylinders, Kodai Math. J. 11 (1988), 295-299.

[6] B.Y. Chen, Null 2-type surfaces in Euclidean space, Proceedings of the Symposium in Honor of Chen-Sung Hsu and Kung-Sing Shih, "Algebra, Analysis and Geometry", National Taiwan Univ., 27-29 June, 1988.

[7] B. Y. Chen ANd S. Lue, Some 2-type submanifolds and applications, Ann. Fac. Sc. Toulouse Math. Ser. V, 9 (1988), 121-131.

[8] S. Nishikawa AND Y. MAEDA, Conformally flat hypersurfaces in a conformally flat Riemannian manifold, Tôhoku Math. J. 26 (1974), 159-168.

[9] P. J. RYAN, Homogeneity and some curvature conditions for hypersurfaces, Tô- 
hoku Math. J. 21 (1969), 363-388.

[10] B. SEgRE, Famiglie di ipersuperficie isoparametrische negli spazi euclidei ad un qualunque numero di dimensioni, Atti. Accad. Naz. Lincei Rend. Cl. Sci. Fis. Mat. Natur. 27 (1938), 203-207.

Departamento de Matemáticas

UNiversidad dE MURcia

CAMPus dE EsPinARDo

30100 Espinardo (Murcia) Spain 